\title{
Virtual conference design: features and obstacles
}

\author{
William Hurst $^{1}$ (D) $\cdot$ Adam Withington $^{2} \cdot$ Hoshang Kolivand ${ }^{3}$
}

Received: 12 January 2021 / Revised: 12 January 2022 / Accepted: 25 January 2022 /

Published online: 3 March 2022

(C) The Author(s) 2022

\begin{abstract}
The Covid-19 pandemic has forced a change in the way people work, and the location that they work from. The impact has caused significant disruption to education, the work environment and how social interactions take place. Online user habits have also changed due to lockdown restrictions and virtual conferencing software has become a vital cog in team communication. In result, a spate in software solutions have emerged in order to support the challenges of remote learning and working. The conferencing software landscape is now a core communication solution for company-wide interaction, team discussions, screen sharing and face-to-face contact. Yet the number of existing platforms is diverse. In this article, a systematic literature review investigation on virtual conferencing is presented. As output from the analysis, 67 key features and 74 obstacles users experience when interacting with virtual conferencing technologies are identified from 60 related open-source journal articles from 5 digital library repositories.
\end{abstract}

Keywords Virtual conferencing $\cdot$ User experience $\cdot$ Covid-19 $\cdot$ User journey mapping

William Hurst

will.hurst@wur.nl

Adam Withington

a.j.withington@ljmu.ac.uk

Hoshang Kolivand

h.kolivand@ljmu.ac.uk

1 Information Technology Group, Wageningen University and Research, Leeuwenborch, Hollandseweg 1, $6706 \mathrm{KN}$ Wageningen, Netherlands

2 Engineering and Technology, Liverpool John Moores University, Byrom Street, Liverpool L3 3AF, UK

3 Department of Computer Science, Liverpool John Moores University, Byrom Street, Liverpool L3 $3 \mathrm{AF}, \mathrm{UK}$ 


\section{Introduction}

In April 2020, 46.6\% of employees were estimated to be working from home during the first wave of the UK Covid-19 pandemic [9]. An increase in digital meetings became a feature of the 2020 work environment with a growing role for virtual conferences [52] and a new range tools emerged to support the transition from the workplace to the home setting [10]. Yet, this move towards a conference virtualisation was already in motion prior to the pandemic. For example, as Forbes et al. outline, in the US, workforces have become more distributed in recent years with $55 \%$ of companies already enabled for remote working prior to the pandemic (with $30 \%$ previously working remotely) [7, 27, 59]. Examples have emerged of businesses that have found working from home to be a way of reducing expenditure (e.g. reduced rent and maintenance costs) [61] and improve staff productivity [14], in some cases by up to 77\% [59]. The effect of Covid-19, however, produced an uncalculated sharp increase in remote working within a shorter space of time than expected with the pandemic acting as a catalyst for an already increasing trend. This sharp increase is evident in the Google search patterns from October 2019 to October 2020 displayed in Fig. 1, demonstrating this sudden inflated interest in virtual conferencing solutions. Search terms Virtual Conference and Covid-19 are depicted, where a value of 100 is the peak popularity. A sustained increase in searches for Virtual Conference remains, continuing in an upward trend beyond the first UK lockdown, whereas a Covid-19 searches are later on the decline.

Aside from affecting the collaborative team working environment, the move to virtual conference settings have had a wider impact across multiple sectors, with corporations unable to showcase products and innovations within expos. Similarly, with universities unable to conduct standard student open days, they have adjusted by means of virtual solutions. For example, TU Delft recreated their campus using Minecraft ${ }^{1}$ to produce a virtualisation of their university grounds to enable students to remotely explore the environment and gain a more indepth impression of the student setting $[11,16] .^{2}$ Other universities have also employed this approach, with a list of virtual tours and videos collated and presented by the Universities and Colleges Admissions Service (UCAS) [68]. As of November 2020, a total of 122 UK-based universities adopted a virtual campus methodology (e.g. interactive maps with 360-degree video capture to create a virtual tour environment, or high-quality video production tours). Yet, so far, there is no unified virtual conference solution employed for product, location or exposition showcasing.

Creating the ideal virtual environment in which participants can communicate effectively remains a prevalent challenge for designers. The notion of culture and having a physical meeting place is something virtual conferences will always struggle to replace. One potential solution to this is to produce ultra-realistic virtual conference environments in $3 \mathrm{D}$, providing a more augmented experience using game engine technologies coupled with headsets and other hardware solutions. To-date, 3D-virtual applications have been proven to be an effective metric for showcasing digital heritage [12] for use with virtual tourism-based applications in particular. Digitising cultural heritage provides sustainability, a way to engage with the public for the sharing of knowledge and virtual tourism opportunities. It is possible that this technology (i.e., game engines) can cater for close-to-real-life environment and interactions. Yet, access to $3 \mathrm{D}$ models and digital content is often a limitation, affecting the quality of the

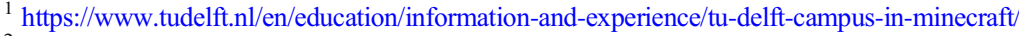

2 https://www.educationandlearning.nl/news/minecraft-campus-update-upcoming-features
} 


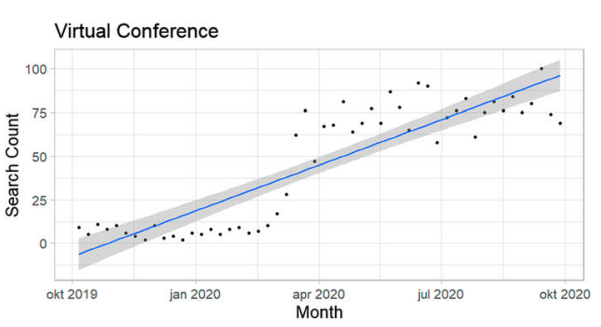

(a)

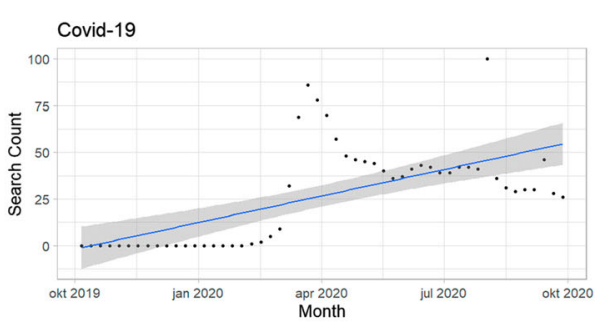

(b)

Fig. 1 Google Trends from Oct 19 to Oct 20 with a depicting virtual conference searches and b Covid-19 searches

production [12] and future digital conference platforms need to recognise the challenge surrounding 3D asset inclusion in the development of a scalable, and engaging environment. For instance, the quality and level of detail (e.g. polygon count) can reduce functionality, despite achieving impressive realism, with multiple users interacting within the environment. Game assets should adhere to low polygon counts, to reduce render times and increase framerate; particularly in real-time expositions. This approach would also require an extensive Infrastructure as a Service (IaaS) to cater for the real-time engagement with the environment and support the scalability for multiple users. This is confirmed by Zhang et al. and Soltanian et al., who emphasise that a suitable cloud infrastructure network is often an issue for multisource multimedia conference systems $[58,74]$. This has led to researchers adopting existing infrastructures, such as Second Life to implement immersive environments as potential virtual conference solutions. August et al., for example, establish a virtual engineering lab that caters for interactive learning through visualisation and problem solving within the virtual Second Life world [5]; thus bypassing the need for creating a new cloud infrastructure.

Despite the sudden growth in the use of virtual conference technologies, it is essential that emerging virtual conference platforms are developed with full consideration of the features and obstacles regarding their design to meet the varied needs of their users. Therefore, in this article, an investigation into the related open source articles that focus on virtual conference solutions is presented. The findings are intended to showcase the features and obstacles associated with existing virtual conference solutions in current literature, specifically from 60 open-source journal articles. The remainder of this article is subsequently organised as follows. Section 2 provides outlines the methodology adopted in for the SLR. Section 3 outlines the results achieved and answers the defined research questions presented in Section 2. The article is concluded in Section 4.

\section{Methodology}

Beyond the need for academic communication and expositions, virtual conferences also serve as suitable training and skills development environments. As Lowe et al. discuss, virtual environments have the potential to be widely adopted for disaster readiness training and education [39]. In their investigation, they document the feasibility of 360 VR technology for adolescent disaster readiness. Yet, the challenge with VR technology, as discussed with by the authors, is that there is a learning curve associated with the use of VR technology headsets and hand controls. For a wider deployment of the technology, for example in a virtual conference setting, all participants would need access to the hardware; meaning there are technological 
barriers. A solution would be to encourage users to attend the virtual conference on their smart phones. Modern-day smart phones are capable of catering for VR technology when coupled with polarised glasses. For example, Tregillus et al. outline that smart phones adaptors enable VR applications to be available for mass audiences. However, there are limitations surrounding interacting with the environment as users are constrained to head movements and are unable to perform hand-based locomotion [64]. Meaning designers must factor in that individuals may not be able to move around and navigate virtual environments. As Mohatta et al. discuss, the future of user interfaces will be governed by hand gestures, and therefore alternative hand gesture techniques are required for integrations with mobile technologies [43].

As highlighted in the related work, there is a willingness to change the way in which conferences take place. There is also a level of existing technology which is capable of supporting this transition. However, a suitable design framework is required for the production of the next generation of virtual conference solutions. In order to contribute to its development, a systematic literature review is conducted on digital conference and digital user experience design works by means of a quality assessment review process. The need for digital inclusion and the growing use of immersive technologies (e.g. augmented and virtual reality) has evolved the variety and functionality of virtual conference solutions. Usability is an indispensable consideration for virtual conference software developers, particularly for cyber learning environments [2]. During a pandemic period that has seen an increasing interest in the development of communication solutions, there must be a corresponding growth in understanding the positive (features) and negative (obstacles) experiences end-users have when using a digital virtual conference product.

\subsection{Systematic literature review}

The systematic literature review (SLR) method adopted for the investigations is an adaptation of model employed by Tummers et al., in [66]. The approach aims to assess available research relevant to the investigation by undertaking six steps within a defined protocol, as follows.

\subsubsection{Research questions}

Based on the related works investigation in 2.1, the investigation will consider all domains in which virtual conferences are used. Specifically, the following questions are identified for the SLR process: Q1: What are the features and obstacles of current virtual conferencing platforms; Q2: What are the user experience considerations? and Q3: What are the digital considerations for $3 D$ virtual conference applications?

\subsubsection{Search strategy}

A systematic search is conducted, focusing on open-source articles available in the IEEE Xplore, MDPI, Elsevier, Springer, Wiley digital libraries (ACM was also considered as a digital library source but was eliminated as, at the time of writing this article, it was not possible to filter articles by open access). The following search queries are conducted. 1) "Virtual Conference (or Digital Conference, or Digital/Virtual Workshop"; 2) "Digital User Experience"; and 3) "3D Conferencing (or Immersive Conferencing)". Due to technology developments within the virtual conferencing domain, only articles from 2016 onwards are considered in the search results. The following pseudocode outlines the search queries employed. 
((((((“All Metadata” : Virtual Conference) OR"All Metadata” : Digital Conference) OR"All

Metadata" : Virtual Expo) OR $\}$ All Metadata $\}$ : Digital Expo) OR $\}$ All Metadata $\}$ : Virtual Workshop $(1)$

OR \}All Metadata\} : Digital Workshop)

(((“All Metadata” : Digital) OR“All Metadata” : Virtual) AND“All Metadata” : User Experience)

((“All Metadata" : 3D OR Immersive) AND“All Metadata" : Conferencing)

\subsubsection{Study selection criteria}

As outlined in Table 1, the selection criteria are applied by a combination of filtering the search then examining the meta data accompanying the publications, reading the title, abstract and conclusion. For Search Criteria 3 (SC3), some search results generated overview documents after the first two stages (e.g. conference or workshop proceedings overviews, or introductions to special issues in journals) rather than full journal articles. This required manual exclusion.

For Search Criteria 4 (SC4), research articles that provide a tool to enable virtual conferencing, even in a one-to-one capacity, are also included (e.g. virtual psychiatry [49], virtual learning [60] etc.). Figure 2 displays the articles selected for quality assessment per search query (i.e. 1-3 listed in Section 2.2.2 above) for each of the digital libraries.

\subsubsection{Quality assessment}

Following SC4, 72 studies are included for the quality assessment process, which is a manual procedure involving reading each of the publications and scoring by means of the quality criteria detailed in Table 2. Points are assigned to the article for providing a valid study (QA1), having clear documentation of methodology (QA2), clear documentation of findings (QA3), conclusion relates to study aims (QA4), overall quality for example clarity, within scope, valid and reliable results (QA5) and relevance to the investigation (QA6).

For the scoring of the criteria, the grading system employed by Tummers et al., in [66] is adopted. In this approach, points are awarded to each criteria on a scale of 1, 0.5 and 0 ; with 1 referring to the highest and 0 the lowest. A score of 0.5 is given if a criteria is somewhat met. As in [66], articles with a total score of $<3$ were excluded from the data extraction and synthesis stages. This meant that 12 studies are excluded prior to the data extraction phase. The selection criteria filtering and quality assessment count is displayed in Tables 3, 4 and 5.

Table 1 Selection Criteria (SC)

\begin{tabular}{ll}
\hline Code & Criteria \\
\hline SC1 & Year (published 2016 onwards). \\
SC2 & Journal Article \\
SC2.1 & Full Text \\
SC2.2 & Written in English \\
SC2.3 & Open Access \\
SC3 & Provides a valid study (e.g. not a foreword or introduction document etc.) \\
SC4 & Related to digital virtual conferencing \\
\hline
\end{tabular}




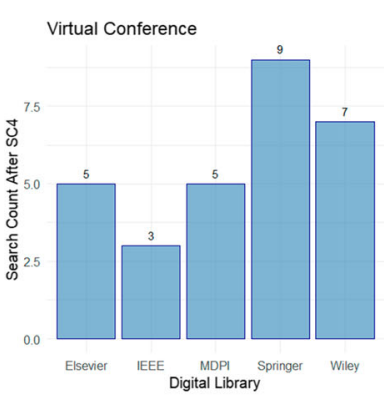

(a)

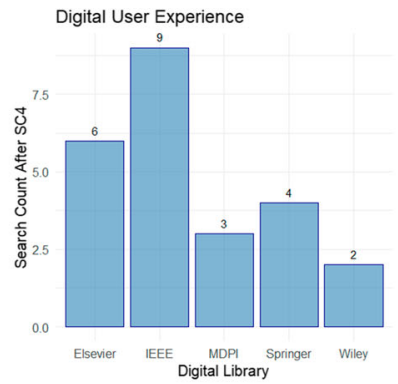

(b)

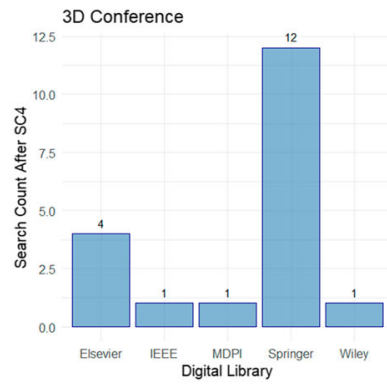

(c)

Fig. 2 Articles Selected for Quality Assessment a Virtual Conference, b Digital User Experience and c 3D Conferencing

\subsubsection{Data extraction}

The data extraction process involves reading the 60 articles, graded by the quality distribution score, of which a distribution of the papers by score is displayed in Fig. 3. The selected papers are used to extract key features and obstacles relating to virtual conference and user experience design from the manuscripts. The data extracted relates to techniques employed, obstacles encountered and assists with answering the research questions. The collated data also caters for understanding and evaluating any emerging trends/patterns in the research. In order to undertake the data extraction process, a structured database is employed (in which articles are assigned a unique identifier) to ensure that the same data aspects are extracted from each of the research articles. Table 6 presents a sample of the dataset constructed following the extraction process (the actual dataset has high granularity). The data extraction process uncovered recurring trends (further outlined Section 3), 152 features and 146 obstacles.

\subsubsection{Data synthesis}

As in [66], the data synthesis process involves the collation of information ascertained during the data extraction process. As the manuscripts often have different terms for the same features, (e.g. computer-mediated communication [65] is within the virtual conference umbrella term), this process involves the use of collating synonyms and deciding on overarching concepts in order to group the features together despite the variations. In total, of the 152 features identified, the data synthesis process reduces the amount down to 67; with the 146 obstacles reduced to 74 . Each of the features and obstacles are grouped into 8 categories, 3D, Social, Multimedia, Data and AI, Competition, Structure, Education and Interaction. A breakdown of the features and obstacles per category is displayed in Fig. 4.

Table 2 Quality Assessment

\begin{tabular}{ll}
\hline Code & Criteria \\
\hline QA1 & Defined and valid study \\
QA2 & Clear documentation of methodology \\
QA3 & Clear documentation of findings \\
QA4 & Does the conclusion relate to study aims. \\
QA5 & Quality (e.g. journal ranking) \\
QA6 & Discusses/Implements Virtual Conferencing Solutions \\
\hline
\end{tabular}


Table 3 Virtual Conference Search

\begin{tabular}{lllllll}
\hline Digital Library & After Search & SC1 & SC2 & SC3 & SC4 & QA \\
\hline IEEE & 291,510 & 64,784 & 642 & 40 & 3 & 3 \\
MDPI & 239 & 209 & 209 & 209 & 5 & 3 \\
Elsevier & 1876 & 1577 & 76 & 76 & 5 & 4 \\
Springer & 1748 & 1122 & 64 & 52 & 9 & 6 \\
Wiley & 22,626 & 6272 & 323 & 283 & 7 & 7 \\
Total & 317,999 & 73,964 & 1314 & 660 & 29 & 23 \\
\hline
\end{tabular}

\section{Results}

This section first covers the main statistics concerning the 60 articles assessed in the SLR process. The section then moves on to answer the research questions identified in 2.2.1.

\subsection{General statistics}

The resulting 60 studies identified following the QA process, are presented in Table 7, with the year-wise distribution of the work displayed in Fig. 5. A higher number of open-access virtualconference related works have been published in 2020 when compared with previous years.

Figure 6 presents a visualisation of the publication domains, following the quality analysis process. The QA process produces 8 domains in which company-wide interaction, team discussions, screen sharing and face-to-face contact research takes place. In some cases, the theme of the article is for the betterment of virtual conferencing specifically; however, if this is the case the article is categorised within the IT/Software category. The Education, Healthcare and IT/Software research domains contained the highest number of publications.

Further to Fig. 6, the score breakdown by journal publisher is presented in Fig. 7, which is also grouped by the publication year. The X-axis refers to the QA score for articles that scored 3 or above (the score distribution is outlined in Section 2, Fig. 3), and the y-axis details the digital repository where the article can be found.

As the graph displays, 2020 resulted in a higher number of virtual conference-related publications, but also more variety in the quality analysis. In the following section, the research questions outlined in Section 2.1.1 are addressed by means of a discussion into the underlying themes in the articles, in which features and obstacles were identified (as in line with the SLR standard, such in the work as by Tummers et al.).

Table 4 Digital User Experience Search

\begin{tabular}{lllllll}
\hline Digital Library & After Search & SC1 & SC2 & SC3 & SC4 & QA \\
\hline IEEE & 5293 & 2807 & 136 & 136 & 9 & 9 \\
MDPI & 1226 & 1101 & 1101 & 1101 & 3 & 2 \\
Elsevier & 9169 & 4730 & 867 & 81 & 6 & 6 \\
Springer & 12,003 & 5760 & 259 & 216 & 4 & 3 \\
Wiley & 3888 & 1704 & 104 & 102 & 2 & 2 \\
Total & 31,579 & 16,102 & 2467 & 1636 & 24 & 22 \\
\hline
\end{tabular}


Table 5 3D Conferencing Search

\begin{tabular}{lllllll}
\hline Digital Library & After Search & SC1 & SC2 & SC3 & SC4 & QA \\
\hline IEEE & 175 & 26 & 1 & 1 & 1 & 1 \\
MDPI & 11 & 9 & 9 & 9 & 1 & 0 \\
Elsevier & 3029 & 1375 & 280 & 82 & 4 & 3 \\
Springer & 3720 & 986 & 176 & 125 & 12 & 11 \\
Wiley & 35,067 & 6334 & 435 & 33 & 1 & 0 \\
Total & 42,002 & 8730 & 901 & 250 & 19 & 15 \\
\hline
\end{tabular}

\subsubsection{Q1: What are the features and obstacles of current virtual conferencing platforms?}

The distribution of the 67 features is displayed in Fig. 8, with a full list presented alphabetically in Table 8 organised by the aforementioned categories (with 3D removed for discussion in 3.2.3). In some instances the features identified are ambiguous, and some features identified in a study are then found to be addressed as obstacles in others. For example, scalability identified as a feature in [34, 58], is referenced as an obstacle in other works [32]. This could be as a result of the differences between the technological requirements between 2D and 3D platforms. As some of the works use 3D technologies (including avatars, virtual reality and immersion) where scalability is an inherent challenge.

Flexibility is listed as a fundamental feature for the structure of the virtual conference. However, the exact specification of what flexibility refers to is ambiguous. Sweetman et al. for example, discuss that accommodating diverse and evolving student scenarios is beneficial [60], and this could be classed as a flexibility feature. Other works document flexibility as a necessity without detailed elaboration [65, 69]. However, features such as bespoke, hybridised, blended learning, collaborative and autonomous may provide an insight into the need for flexibility. Furthermore, as Schouten et al. discuss, there is a need to allow individual users to indicate their own learning styles [54], meaning flexibility is required in the conference platform to customise the learning or communication environments for both the teacher and students [48].

Core features recur throughout many of the articles including, audio, text, content sharing, engagement, free and social interaction. However, some works refer to other more unique features, such as competition, autonomy and reward systems. As Sardi et al. discuss, by introducing competition and a reward system (e.g. badges and points) and leader boards,

Quality Assessment Scores

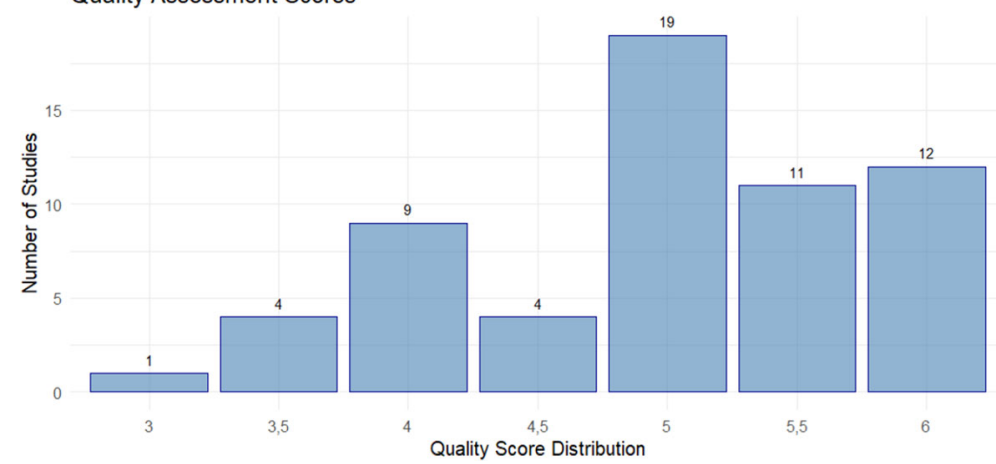

Fig. 3 Quality Score Distribution 
Table 6 Data Extract Sample

\begin{tabular}{|c|c|c|c|c|c|c|}
\hline $\begin{array}{l}\text { Article } \\
\text { ID }\end{array}$ & Keywords & Tools & $\begin{array}{l}\text { UX } \\
\text { Points }\end{array}$ & $\begin{array}{l}\text { Targeted } \\
\text { Domain }\end{array}$ & Obstacles & Features \\
\hline $\mathrm{VC} 1$ & $\begin{array}{l}\text { Covid-19, disseminate, } \\
\text { collaborations, feedback }\end{array}$ & $\begin{array}{l}\text { WebEx, Web } \\
\text { 2.0, Zoom }\end{array}$ & Lean & Medical & $\begin{array}{l}\text { Multiple } \\
\text { technolo- } \\
\text { gies }\end{array}$ & Scalable \\
\hline
\end{tabular}

effective solutions for learning-based environments [53] the need for continuous feedback in other domains could also be made possible.

Regarding extraction of the obstacles found in the articles, examples include synchronous delivery [4], cybersickness [3], immersion quality [25], scalability [32] and avatar personalisation [26]. All obstacles identified in the SLR for all virtual meeting contexts, are presented alphabetically in Table 9, again organised into the aforementioned categories.

\subsubsection{Q2: What are the user experience considerations?}

41 user experience considerations are identified in the literature survey, as outlined in Table 10, again, presented alphabetically. Two notable recurring considerations are the need for better presence (a feeling of being there in a perceptible external world around the self) [37] during a virtual conference session and the lack of being able to see physical gestures (and eye contacts) [8]. For example, as Cai et al. discuss, social cues affect remote communication, and an additional camera may be appropriate in order to provide a viewing perspective, which would allow for the conveyance of mutual gesture cues [8].

In addition to the above considerations, 74 obstacles are identified. The distribution of the obstacles across the 8 categories outlined in Section 2.2.6 is displayed in Fig. 9. The highest volume of obstacles is related to social considerations. This would be in line with the user experience design issues identified above, which outline the need for better social cue integration into virtual conference platforms.

Table 10 details the full list of obstacles. Digital inclusion is a recurring challenge within the works identified. Access to technology and the skills required to operate tools (e.g. haptic control devices [3]) and equipment (e.g. high-speed internet [5] and VR-gear) are natural boundaries for the end-user when working with virtual conferencing solutions. However, technology must also cater for users with disabilities such as deuteranopia, autism, intellectual disability, emotional disturbance, etc. [24]. This is more so the case when the technology involves communication in a virtual conference when VR is employed or interacting with screen-based 3D conference environments.

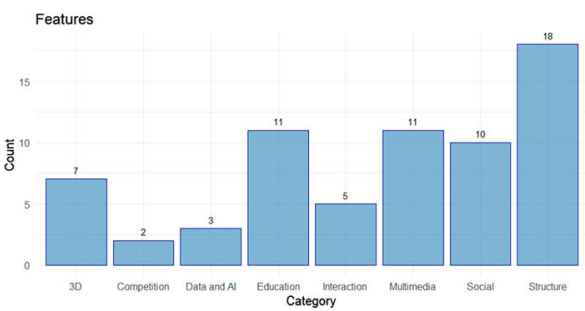

(a)

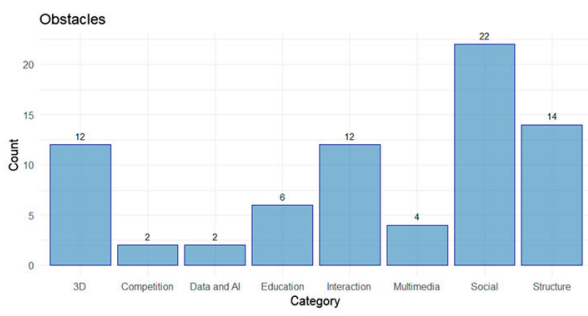

(b)

Fig. 4 Category Distribution for a Features and b Obstacles 
Table 7 Primary Studies Following QA in Order of Search

\begin{tabular}{llllll}
\hline$[31]$ & {$[72]$} & {$[58]$} & {$[19]$} & {$[30]$} & {$[1]$} \\
{$[20]$} & {$[32]$} & {$[37]$} & {$[8]$} & {$[22]$} \\
{$[75]$} & {$[61]$} & {$[3]$} & {$[52]$} & {$[41]$} & {$[50]$} \\
{$[65]$} & {$[6]$} & {$[25]$} & {$[57]$} & {$[46]$} & {$[60]$} \\
{$[26]$} & {$[33]$} & {$[56]$} & {$[44]$} & {$[54]$} & {$[21]$} \\
{$[53]$} & {$[42]$} & {$[18]$} & {$[67]$} & {$[62]$} & {$[38]$} \\
{$[29]$} & {$[34]$} & {$[73]$} & {$[4]$} & {$[13]$} & {$[49]$} \\
{$[55]$} & {$[47]$} & {$[17]$} & {$[28]$} & {$[69]$} & {$[23]$} \\
{$[40]$} & {$[12]$} & {$[76]$} & {$[63]$} & {$[51]$} & {$[71]$} \\
{$[48]$} & {$[74]$} & {$[36]$} & & {$[45]$} \\
\hline
\end{tabular}

\subsubsection{Q3: What are the digital considerations for 3D virtual conference applications?}

Regarding 3D conferencing, completed 3D models are identified as a critical issue. Incomplete 3D models can produce a disturbing experience for users [18]. Also within a 3D-based environment, puppeteering may be required to allow the user to control their virtual avatar [32] when negotiating the virtual environment. For example, using the keyboard and mouse separately to move different body parts would allow the user to integrate physical gestures when communicating.

However, access to technology for the conference provider is also a challenge. When developing a 3D conference solution a cloud-based infrastructure is inevitable to provide scalability and address the need for real-time interaction. For that reason, 5 of the 60 works reference existing tools such as Second Life for the virtual environment applications $[5,13,26$, $37,62]$. In this case, the backend infrastructure and $3 \mathrm{D}$ world are already created, meaning the developer is benefitting from the use of third party technology. However, this also means that control over the environment is limited and customisation may be restricted. This issue of lack of control is identified as a potential consideration by Ding et al. [17]. Table 11 presents the full list of 3D considerations divided into features and obstacles.

VR is a common approach for next-generation 3D virtual conference solutions, and 20 of the 60 articles reference the technology as a potential education, training and communication solution moving forwards. However, Liu et al. discuss that negative immersive experiences can emerge when a user moves too quickly in VR [35] and other challenges, such as cybersickness, need to be addressed before wider deployment [3]. Yet, 3D and VR are remain prominent technologies for immersive training, and cultural heritage communication applications [12] particularly.

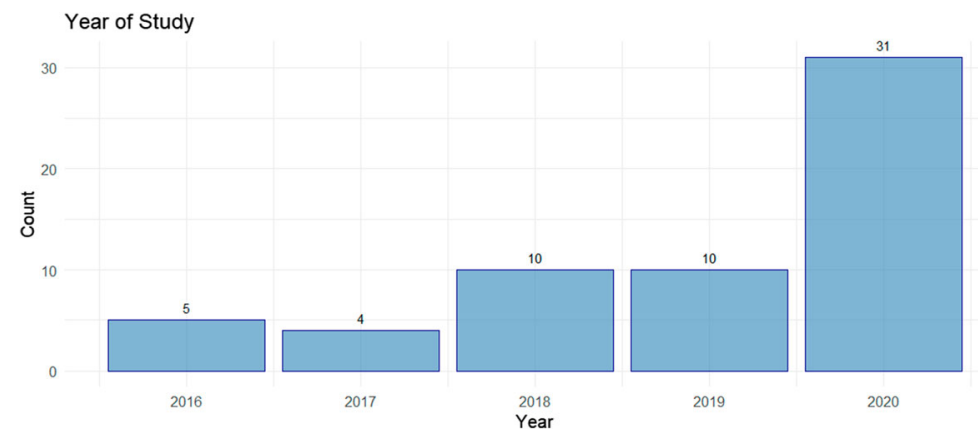

Fig. 5 Year-wise Distribution of 60 Studies 
Domain of Studies

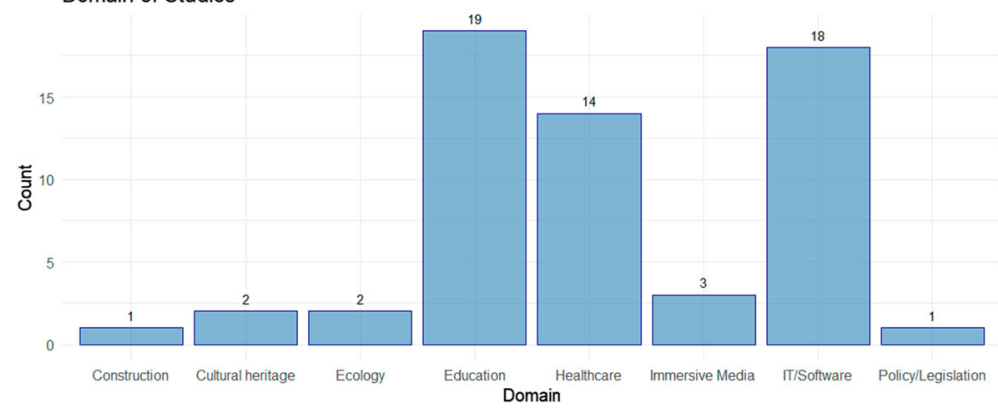

Fig. 6 Domain of Studies

\subsection{Discussion}

Section 3.1 provides a statistical overview based on an SLR analysis, concentrating the search on IEEE Xplore, MDPI, Elsevier, Springer, Wiley digital libraries. To the best of our knowledge, this article presents the first SLR analysis of virtual conference solutions that integrates 3D into the investigation. With a total of 391,580 articles identified, 60 were then selected for data extraction and discussion based on a comprehensive selection process and quality assessment. The subsequent analysis of the 60 articles provides 67 features and 72 obstacles, which can be of value for virtual conference technology developers in creating their software applications. Both the features and obstacles were divisible into 8 categories: 3D, Competition, Data and AI, Education, Interaction, Multimedia, Social and Structure.

However, one further contribution to knowledge is discussion of the recurring underlying themes within the 60 articles. One of the most prominent is Covid-19, which is often argued as a motivator or catalyst for the research taking place. As discussed in the introduction, the trend towards working from home and virtual conference solutions to facilitate this was already under-way pre-pandemic, but the pandemic has acted as a catalyst speeding up this process.

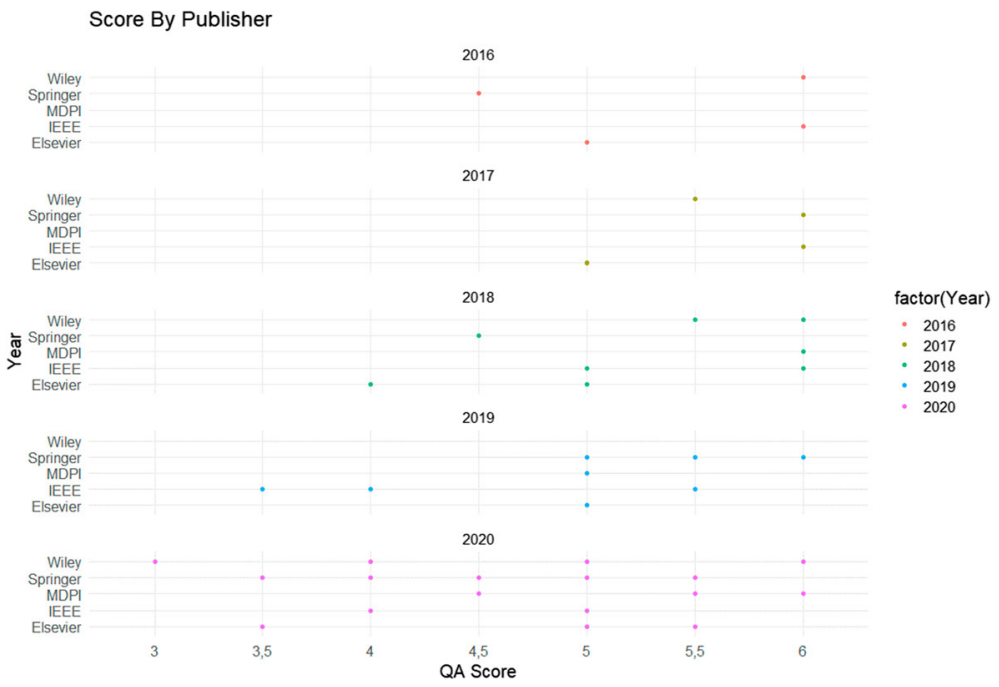

Fig. 7 Score by Publisher 


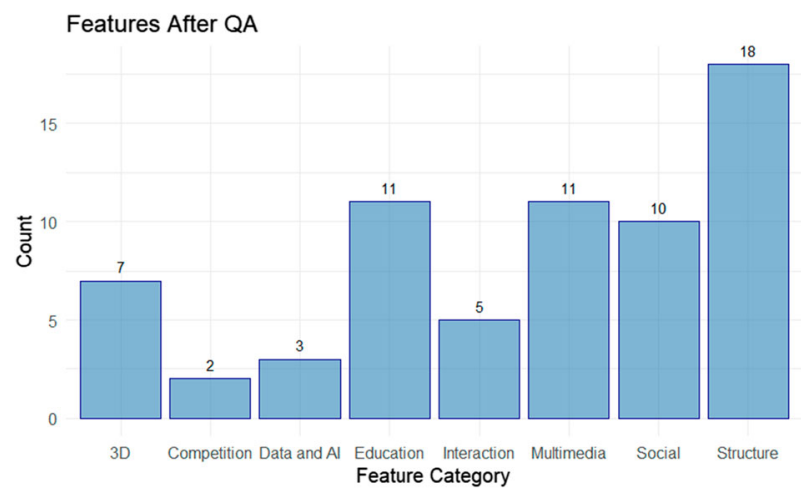

Fig. 8 Feature Count after QA

Yet, covid-19 has created a somewhat prominent line of focus within the articles in the present period (and its peculiar requirements and problems). To name a few examples, Lamming et al. discuss the use of an online seminar series as a result of the Covid-19 pandemic affecting regional and national meetings [31]; Milovanović et al. outline that Covid-19 has brought considerable challenges resulting in the need for the emergency design of education material [42]; and Rubinger et al. detail that the Covid-19 pandemic has created a need to maximise communication within the medical and scientific community [52]. Out of the 60 articles, 15 reference Covid-19. This could be the core reason for why a higher number of articles are present from 2020 in the search results compared with the previous four years. Again, the domain of the articles is relating majorly to healthcare, education and IT, which would be logical due to the impact Covid-19 has had on education, training and the medical infrastructure.

Other recurring trends include virtual interviews, virtual training, digital reliance, gamification and social connections. Culture is also a recurring keyword within the articles for two reasons, some of the articles are related directly to digital cultural heritage applications [12]. However, in other cases the works refer to the need to virtual conference tools to embrace a solution for the lack of workplace culture when working from home, particularly for medicine education [20]. It is, of course, a challenge to recreate a workplace culture within a digital setting, or recreate a learning culture that a student would find typically in a physical classroom environment. Yet, the investigation uncovered features that are beneficial to the virtual digital conference experience. Whilst this (the need for the creation of culture) was an unexpected finding, it would a logical consideration as the need for meeting in person, for example at a physical international conference, has benefits beyond the access the knowledge sharing. Considerations, such as this, may be easy to overlook when developing a virtual conference application, but they are crucial insights for both global virtual teams literature and virtual teams or those in educational and management positions, which emphasises the importance of regular SLR investigations that survey the existing landscape.

The authors also emphasise that the aims and findings depicted in the paper are not intended to promote the use virtual conferencing solutions over other approaches or dissuade from their use, but rather showcase the existing features and obstacles 


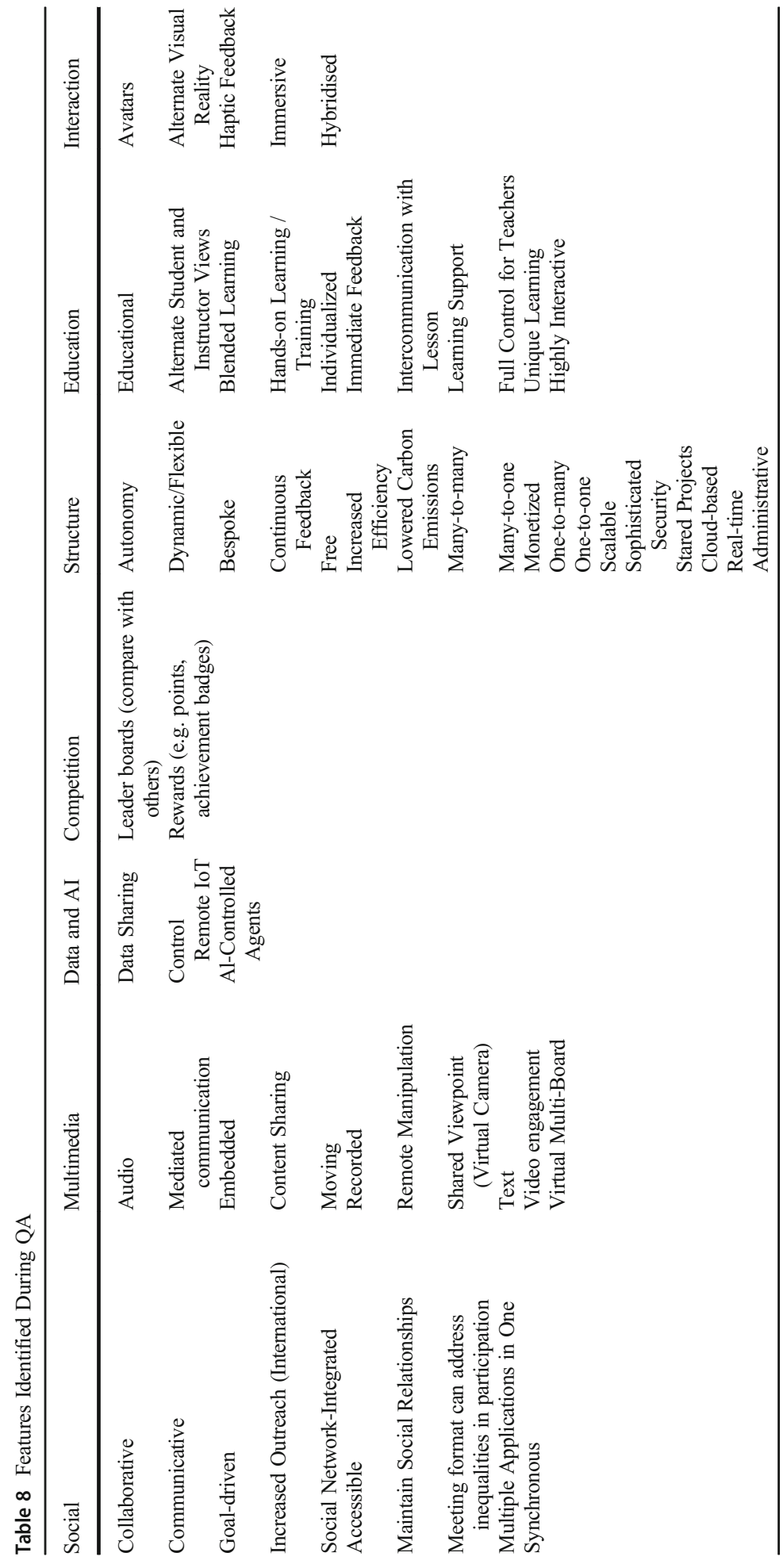




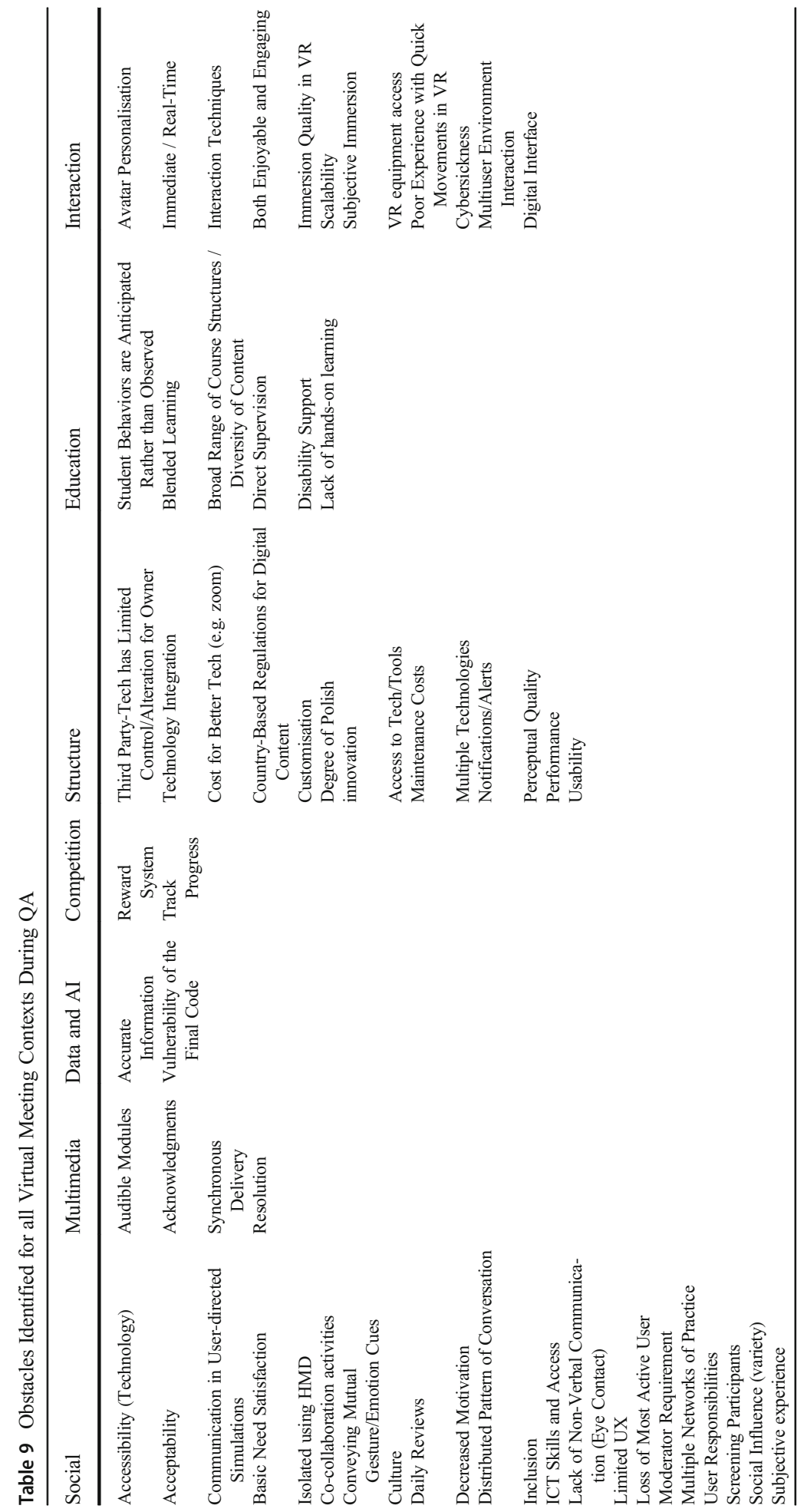


Table 10 User Experience Considerations

\begin{tabular}{|c|c|c|c|}
\hline Adaptable & $\begin{array}{l}\text { Ease of } \\
\text { interaction }\end{array}$ & Incompleteness & $\begin{array}{l}\text { Reduced Waiting Time on Start- } \\
\text { up }\end{array}$ \\
\hline Ambient Intelligence & Efficient & Interactive & Regions of Interest Areas \\
\hline $\begin{array}{l}\text { Awareness } \\
\text { tool/interface }\end{array}$ & Empathy & Intimacy & Reliable \\
\hline Cloud Architecture & $\begin{array}{l}\text { Environmentally } \\
\text { Conscious } \\
\text { Design }\end{array}$ & Lean & Responding to User Needs \\
\hline Code of conduct & Extended Reality & Mobile & Structured \\
\hline Computer-Guided & Gesture Detection & Multimodal & Tactile \\
\hline Decentralised & $\begin{array}{l}\text { High Computer } \\
\text { Memory } \\
(1-3 \mathrm{~GB})\end{array}$ & Presence & Untethered \\
\hline $\begin{array}{l}\text { Design Style that } \\
\text { Reuses Web } \\
\text { Technologies }\end{array}$ & $\begin{array}{l}\text { High-Speed } \\
\text { Internet } \\
\text { Connection }\end{array}$ & $\begin{array}{l}\text { Puppeteered (using users' } \\
\text { keyboard, mouse, or } \\
\text { controller inputs) }\end{array}$ & User-Friendly Interface \\
\hline Digitally Inclusive & $\begin{array}{l}\text { Human Computer } \\
\text { Interaction }\end{array}$ & Quality of Experience & Visual Attention \\
\hline Duration & Immediacy & Realism of the image & $\begin{array}{l}\text { Well-balanced (synergy between } \\
\text { representation and } \\
\text { experience) }\end{array}$ \\
\hline
\end{tabular}

associated with existing software as is found in current open access literature only. This is, of course, also a limitation of the work, as it may be the case that some prominent paid-for articles have unique features or obstacles that would benefit this investigation. Yet, the adoption of the open-access approach in this article is intentional in order to make this research repeatable and, similarly, the findings discussed from the related articles available to as wide an audience as possible. This means others will be able to review the examined references. It is also evident that the volume of open-access research articles related to virtual conference applications has increased sufficiently over the last five years to provide a detailed and structured SLR output.

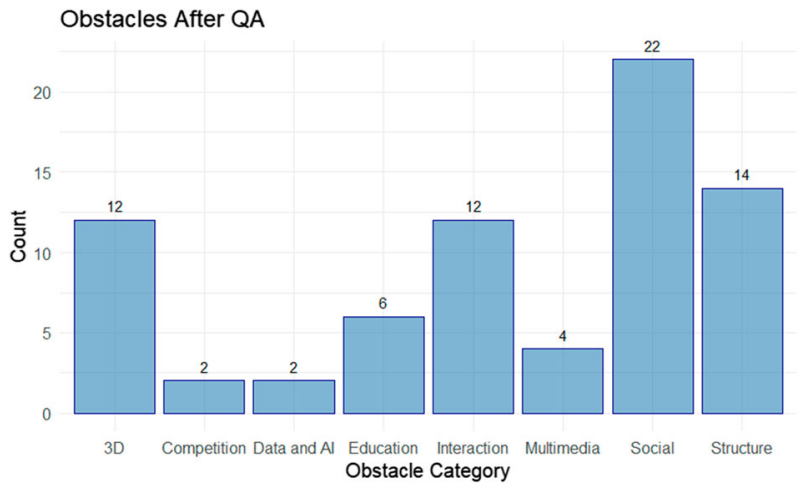

Fig. 9 Count for Obstacles Following QA 
Table 11 Considerations for 3D in virtual conference applications

\begin{tabular}{ll}
\hline Features & Obstacles \\
\hline 360 Degree videos & 3D Content Production \\
Accurate representation of indoor environment & 3D Animation Production \\
Harness BIM & BIM Files Have Large Geometry \\
Digital gaming & Field of View Awareness \\
Digital twin & Continuous Improvement Requirement \\
Virtual humans & Need for dedicated device(e.g. Game Controller) \\
Virtual Reality & Fluidity \\
& Quality and Realism \\
& Scanning Requirements \\
& Texture Parameters \\
& Camera FOV \\
& Decreased Frame Rate \\
\hline
\end{tabular}

\section{Conclusion}

The Covid-19 pandemic has had a disruptive impact in 2020, but it has also created opportunities for virtual conference creators to develop solutions to support remote working, but the pandemic has acted as a catalyst to bring the notion of virtual conferencing more into the mainstream. Virtual conferencing may also remain part of our work environment for the foreseeable future; with authors such as [23] and [21] arguing that virtual conferencing is becoming a preferred solution for those wishing to reduce their carbon footprint, meaning virtual conference development opportunities may extend beyond pandemics as conference participation is an essential aspect for the development of research and creation of novel ideas. Therefore, immersive virtual conferencing may be an ideal solution, if yet a challenge.

In this article, an investigation into the features and obstacles found within virtual conference solutions (both 2D and 3D) are discussed. Academic literature repositories were the primary target of the investigation, however, this could also be expanded to include other sources, such as blogs and other grey literature in future investigations, where there are often software applications not considered in academic publications. In addition, paid-for articles were not part of the SLR investigation, which is a limitation and it is possible that articles suitable for discussion were omitted from the findings. However, an open-access review approach was adopted to cater for repeatability of the research and for direct access to the findings from the articles discussed in the SLR. Our approach also means it is possible to expand the investigation by the inclusion of such articles in future studies. In our future work, we will devise a decision model framework to help others plan and setup a feature-driven conference solution based on the data collated in the SLR presented in this article. Future directions of the research could also include survey-based studies of existing solutions to a wide group of end-users or duplications of the investigation using paid for articles only to provide a comparison of the findings.

\section{Declarations}

Ethical approval This article does not contain any studies with human participants or animals performed by any of the authors.

Informal consent There were no individual participants included in the study. 
Conflict of interest There is no conflict of interest between the authors regarding the manuscript preparation and submission.

Open Access This article is licensed under a Creative Commons Attribution 4.0 International License, which permits use, sharing, adaptation, distribution and reproduction in any medium or format, as long as you give appropriate credit to the original author(s) and the source, provide a link to the Creative Commons licence, and indicate if changes were made. The images or other third party material in this article are included in the article's Creative Commons licence, unless indicated otherwise in a credit line to the material. If material is not included in the article's Creative Commons licence and your intended use is not permitted by statutory regulation or exceeds the permitted use, you will need to obtain permission directly from the copyright holder. To view a copy of this licence, visit http://creativecommons.org/licenses/by/4.0/.

\section{References}

1. Ahrar S, Li DJ, Towles JD, Venook RD (2020) Trace matrix: a framing tool to improve communication and debugging in remote instrumentation lab courses. Biomedical Engineering Education

2. Alomaria HW, Ramasamya V, Kipera JD, Potvin G (2020) A user Interface (UI) and user eXperience (UX) evaluation framework for cyberlearning environments in computer science and software engineering education. Heliyon 6(5):e03917

3. Anwar MS, Wang J, Khan W, Ullah A, Ahmad S, Fei Z (2020) Subjective QoE of 360-degree virtual reality videos and machine learning predictions. IEEE Access 8:148084-148099

4. Arena SL et al. (2020) Web conferencing facilitation within problem-based learning biomedical engineering courses. Biomedical Engineering Education

5. August SE, Hammers ML, Murphy DB, Neyer A, Gueye P, Thames RQ (2015) Virtual engineering sciences learning lab: giving STEM education a second life. IEEE Trans Learn Technol 9(1):18-30

6. Boje C, Guerriero A, Kubicki S, Rezgui Y (2020) Towards a semantic construction digital twin: directions for future research. Autom Constr 114:103179

7. Bump P (2020) 40 remote work stats to know in 2020. [online], Available at: https://blog.hubspot.com/ marketing/remote-work-stats. Accessed Nov 2020

8. Cai M, Tanaka J (2019) Go together: providing nonverbal awareness cues to enhance co-located sensation in remote communication. Human-centric computing and information sciences, volume 9

9. Cameron A (2020) Coronavirus and homeworking in the UK: April 2020. Office for National Statistics, London

10. Case J (2020) Zoom, Microsoft teams, and slack have exploded due to the COVID-19 pandemic. Can they hold onto this growth?. Industry Insights, 1 April.

11. Centre of Education and Learning, 2020. Minecraft Campus Update: Upcoming Features!. [Online], Available at: https://www.educationandlearning.nl/news/minecraft-campus-update-upcoming-features. Accessed Nov 2020

12. Champion E, Rahaman H (2019) 3D digital heritage models as sustainable scholarly resources. Sustainability 11(8):2425

13. Cheng A, Kolbe M, Grant V (2020) A practical guide to virtual debriefings: communities of inquiry perspective. Adv Simul 5(18):18

14. Clark B, Chatterjee K, Martin A, Davis A (2019) How commuting affects subjective wellbeing. Transportation 47:2777-2805

15. Clark-Wilson A, Robutti O, Thomas M (2020) Teaching with digital technology. ZDM 52:1223-1242

16. Delft T (2020) TU Delft Campus in Minecraft. [Online], Available at: https://www.tudelft.nl/en/education/ information-and-experience/tu-delft-campus-in-minecraft/. Accessed 17 Dec 2020

17. Ding Y, Li Y, Cheng L (2020) Application of internet of things and virtual reality Technology in College Physical Education. IEEE Access 8:96065-96074

18. Dong H, Ganz A (2019) Cost efficient virtual environment generation framework using annotated panoramic videos. IEEE Access 7:121024-121036

19. Doumanoglou A (2018) Quality of experience for 3-D immersive media streaming. EEE Trans Broadcast 64(2):379-391

20. Everett AS, Strickler S, Marcrom SR, McDonald AM (2020. Students' perspectives and concerns for the 2020 to 2021 radiation oncology interview season. Advances in radiation oncology, Issue In Press

21. Ewijk S v, Hoekman P (2020) Emission reduction potentials for academic conference travel. J Ind Ecol 
22. Faraji S, Valizadeh S, Sharifi A, Shahbazi S, Ghojazadeh M (2020) The effectiveness of telegram-based virtual education versus in-person education on the quality of life in adolescents with moderate-to-severe asthma: a pilot randomized controlled trial. Nursing Open 7(6):1691-1697

23. Fraser H, Soanes K, Jones SA, Jones CS, Malishev M (2016) The value of virtual conferencing for ecology and conservation. Conserv Biol 31(3):540-546

24. Frederick JK, Raabe GR, Rogers VR, Pizzica J (2020) Advocacy, collaboration, and intervention: a model of distance special education support services amid COVID-19. Behav Anal Pract 13:748-756

25. García-Valle G, Ferre M, Breñosa J, Vargas D (2018) Evaluation of presence in virtual environments: haptic vest and User's haptic skills. IEEE Access 6:7224-7233

26. Horne M, Hill A, Murells T, Ugail H, Irving, Chinnadorai R, Hardy M (2020) Using avatars in weight management settings: a systematic review. Internet Interv 19:110295

27. Insights F (2019) The visual advantage: harnessing video-led unified communications to drive a more agile, connected and effective culture. Forbes, Jersey City

28. Janssen A, Passlick J, Cardona DR, Breitner MH (2020) Virtual assistance in any context. Bus Inf Syst Eng 62:211-225

29. Johnson D, Deterding S, Kuhn KA, Staneva A, Stoyanov S, Hides L (2016) Gamification for health and wellbeing: a systematic review of the literature. Internet Interv 6:89-106

30. Kim S, Billinghurst M, Kim K (2020) Multimodal interfaces and communication cues for remote collaboration. J Multimodal User Interfaces 14:313-319

31. Lamming DW, Carter CS (2020) Maintaining a scientific community while social distancing. Transl Med Aging 4:55-59

32. Latoschik ME, Kern F, Stauffert JP, Bartl A, Botsch M, Lugrin JL (2019) Not alone Here?! Scalability and user experience of embodied ambient crowds in distributed social virtual reality. IEEE Trans Vis Comput Graph 25(5):2134-2144

33. Leonidis A et al (2020) CognitOS board: a wall-sized board to support presentations in intelligent environments. MDPI Technologies 8(4):66

34. Liddle $\mathrm{J}$ et al (2020) Connecting at local level: exploring opportunities for future design of technology to support social connections in age-friendly communities. MDPI Environmental Research and Public Health 17(15):5544

35. Liu, W.-H. \& Chiang, Z.-H., 2018. Information visualization- in regarding to customer journey map in a three-dimensions format. Jeju, s.n.

36. Liu D, An P, Ma R, Zhan W, Ai L (2018) Scalable omnidirectional video coding for real-time virtual reality applications. IEEE Access 6:56323-56332

37. Lombardo, E., Guion, C. \& Keller, J., 2016. Study of a virtual conference in a mirror world with avatars and HMD. Part of the lecture notes in computer science, pp 330-338.

38. Lortie CJ (2020) Online conferences for better learning. Ecol Evol 10(22):12442-12449

39. Lowe J, Peng C, Winstead-Derlega C, Curtis H (2020) 360 virtual reality paediatric mass casualty incident: a cross sectional observational study of triage and out-of-hospital intervention accuracy at a national conference. Journal of the American College of Emergency Physicians Open 1(5):974-980

40. Machidon OM, Duguleana M, Carrozzino M (2018) Virtual humans in cultural heritage ICT applications: a review. J Cult Herit 33:249-260

41. Mallik B, Sheikh-Akbari A, Kor A-L (2019) Mixed-resolution HEVC based Multiview video codec for low bitrate transmission. Multimed Tools Appl 78:6701-6720

42. Milovanović A et al (2020) Transferring COVID-19 challenges into learning potentials: online workshops in architectural education. MDPI Sustain 12(17):7024

43. Mohatta, S. et al., 2017. Robust hand gestural interaction for smartphone based AR/VR applications. Santa Rosa, s.n.

44. Niner HJ, Johri S, Meyer J, Wassermann SN (2020) The pandemic push: can COVID-19 reinvent conferences to models rooted in sustainability, equitability and inclusion? Socio-Ecological Practice Research 2:253-256

45. Nooren P, Gorp N v, Eijk N v, Fathaigh RÓ (2018) Should we regulate digital platforms? A new framework for evaluating policy options. Policy Internet 10(3):264-301

46. Oliver S (2019) Communication and trust: rethinking the way construction industry professionals and software vendors utilise computer communication mediums. Visualization in Engineering 7

47. Osimani F, Stecanella B, Capdehourat G, Etcheverry L, Grampín E (2019) Managing devices of a one-toone computing educational program using an IoT infrastructure. MDPi Sensors 19(1)

48. Philippe S, Souchet AD, Lameras P, Petridis P (2020) Multimodal teaching, learning and training in virtual reality: a review and case study. Virtual Reality and Intelligent Hardware 2(5):421-442

49. Pickersgill M (2018) Digitising psychiatry? Sociotechnical expectations, performative nominalism and biomedical virtue in (digital) psychiatric praxis. Sociol Health Illn 41(S1):16-30 
50. Rawle M (2017) Improving education and supervision of Queensland X-ray operators through video conference technology: a teleradiography pilot project. J Med Radiat Sci 64(4):244-250

51. Rothe S, Schmidt A, Montagud M, Buschek D, Hußmann H (2020) Social viewing in cinematic virtual reality: a design space for social movie applications. Virtual Reality 25:613-630

52. Rubinger L, Gazendam A, Ekhtiari S, Nucci N, Payne A, Johal H, Khanduja V, Bhandari M (2020) Maximizing virtual meetings and conferences: a review of best practices. Int Orthop 44:1461-1466

53. Sardi L, José A, Fernández-Alemán L (2017) A systematic review of gamification in e-health. J Biomed Inform 71:31-48

54. Schouten DGM, Smets NJJM, Driessen M, Fuhri K, Neerincx MA, Cremers AHM (2017) Requirements for a virtual environment to support the social participation education of low-literates. Univ Access Inf Soc 16 : 681-698

55. Silva JN, Southworth M, Raptis C, Silva J (2018) Emerging applications of virtual reality in cardiovascular medicine. JACC: Basic to Translational Science 3(3):420-430

56. Simiscuka AA, Markande TM, Muntean G-M (2019) Real-virtual world device synchronization in a cloudenabled social virtual reality IoT network. IEEE Access 7:106588-106599

57. Smigelski M, Movassaghi M, Small A (2020) Urology virtual education programs during the COVID-19 pandemic. Education 21:50

58. Soltanian A, Belqasmi F, Yangui S, Salahuddin MA, Glitho R, Elbiaze H (2018) A cloud-based architecture for multimedia conferencing service provisioning. IEEE Access 6:9792-9806

59. Stone K (2020) The state of video conferencing in 2020 [50 statistics]. [online], Available at: https://getvoip. com/blog/2020/07/07/video-conferencing-stats/. Accessed Nov 2020

60. Sweetman DS (2020) Making virtual learning engaging and interactive. BioAdvances 1-22

61. Syamimi A, Gong Y, Liew R (2020) VR industrial applications-a Singapore perspective. Virtual Reality \& Intelligent Hardware 2(5):409-420

62. Taylor MSCMC (2020) Using virtual worlds as a platform for collaborative meetings in healthcare: a feasibility study. BMC Health Serv Res 20:442

63. Tibaná-Herrera G, Fernández-Bajón MT, Moya-Anegón FD (2018) Categorization of E-learning as an emerging discipline in the world publication system: a bibliometric study in SCOPUS. Int J Educ Technol High Educ 15

64. Tregillus S, Folmer E (2016). VR-STEP: walking-in-place using inertial sensing for. New York, s.n.

65. Trust T, Krutka DG, Carpenter JP (2016) "Together we are better": professional learning networks for teachers. Comput Educ 102:15-34

66. Tummers J, Kassahun A, Tekinerdogan B (2019) Obstacles and features of farm management information systems: a. Comput Electron Agric 157:189-204

67. Turner S, Pham T, Robledo K, Turner S, Brown C, Sundaresan P (2020) Rapid adaptation of Cancer education in response to the COVID-19 pandemic: evaluation of a live virtual statistics and research skills workshop for oncology trainees. J Cancer Educ

68. UCAS (2020) Virtual Tours. [online], Available at: https://www.ucas.com/undergraduate/what-and-wherestudy/open-days-and-events/virtual-tours. Accessed Nov 2020

69. Vielma K, Bre EM (2020) Using evaluative data to assess virtual learning experiences for students during COVID-19. Biomedical Engineering Education.

70. Wilde P (2015) Flying less: reducing Academia's carbon footprint. [online] Available at: https:// academicflyingblog.wordpress.com/. Accessed 11 Nov 2020

71. Wilkerson B, Aguiar A, Gkini C, Czermainski de Oliveira I, Lunde Trellevik LK, Kopainsky B (2020) Reflections on adapting group model building scripts into online workshops. Syst Dyn Rev 36(3):358-372

72. Zhang Y, Fei G (2019) Overview of 3D scene viewpoints evaluation method. Virtual Reality \& Intelligent Hardware 1(4):341-385

73. Zhang S, Zhang S (2019) A novel human-3DTV interaction system based on free hand gestures and a touch-based virtual Interface. IEEE Access 7:165961-165973

74. Zhang W, Zhang X, Shi H (2018) MMCSACC: a multi-source multimedia conference system assisted by cloud computing for smart campus. IEEE Access 6:35879-35889

75. Zöller N, Morgan JH, Schröder T (2020) A topology of groups: what GitHub can tell us about online collaboration. Technological Forecasting and Social Change 161:120291

76. Zou W, Zhang W, Song J, Yang F, le Callet P (2019) A novel method for assessing the spatial presence of omnidirectional videos: comparing virtual and real world. IEEE Access 7:183405-183413

Publisher's note Springer Nature remains neutral with regard to jurisdictional claims in published maps and institutional affiliations. 